\title{
Modelo para la Evaluación de Programas de Ética Organizacional en Universidades
}

\author{
Valentina Osorio $^{(1)}$ y María del P. Rodríguez ${ }^{(2)}$ \\ (1) Universidad Nacional de Colombia Sede Manizales, Campus la Nubia, Km 4 Vía al Magdalena, \\ Grupo de Investigación Ethos - Bloque Q, Manizales, Caldas - Colombia. (e-mail: vosoriov@unal.edu.co) \\ (2) Universidad Nacional de Colombia Sede Manizales, Facultad de Ingeniería y Arquitectura, \\ Depto. de Ingeniería Industrial, Campus la Nubia, Km 4 Vía al Magdalena, Manizales, Caldas - Colombia. \\ (e-mail: mdrodriguezco@unal.edu.co)
}

Recibido May. 8, 2017; Aceptado Jul. 5, 2017; Versión final Ago. 29, 2017, Publicado Feb. 2018

\begin{abstract}
Resumen
En este artículo se propone un modelo para evaluar programas de ética organizacional implementados en universidades. La metodología utilizada es cualitativa y tiene en cuenta el antecedente de la Universidad Nacional de Colombia, donde existió un programa de ética. La revisión bibliográfica muestra que hay un vacío en el conocimiento sobre evaluación de programas de ética, en general, y en las universidades, en particular. El principal resultado es un modelo de evaluación basado en instrumentos de corte cualitativo y cuantitativo. Se concluye que el modelo propuesto permite evaluar programas de ética organizacional en universidades, dando cuenta no sólo de la ejecución presupuestal y la calidad de las actividades realizadas, sino también de otros aspectos. Entre estos, las percepciones de los grupos de interés en cuanto a la apropiación del código ético y los valores morales, los cambios en las actitudes y comportamientos éticos y el fortalecimiento de la cultura organizacional ética.
\end{abstract}

Palabras clave: ética organizacional; programas de ética; evaluación de programas; universidades; grupos de interés

\section{Model for the Evaluation of Organizational Ethics Programs in Universities}

\begin{abstract}
A model for the evaluation of organizational ethics programs implemented in universities is proposed. The methodology used is qualitative and it considers the results of an ethics program of the Universidad Nacional de Colombia. The literature review shows that there is a knowledge gap about evaluation of ethics programs, in general, and in universities, in particular. The main result is a model of evaluation based in qualitative and quantitative instruments. It is concluded that the proposed model allows evaluating organizational ethics programs in universities, given an account not only of the budget execution and the quality of the activities realized, but also of other factors. Among these are the perceptions of the stakeholders regarding the appropriation of the organizational ethical code and moral values, the changes in ethical attitudes and behaviors and the strengthening of the ethical organizational culture.
\end{abstract}

Keywords: organizational ethics; ethics programs; program evaluation; universities; stakeholders 


\section{INTRODUCCIÓN}

Las organizaciones son la creación más poderosa del ser humano y, sin embargo, también la más peligrosa, pues pueden usarse para la búsqueda del bien común o para el logro de fines egoístas. Por esto, ellas necesitan de la ética, una rama de la filosofía que reflexiona sobre los comportamientos de las personas, a partir del análisis sobre lo que es bueno o malo, correcto o incorrecto. La ética ayuda a que los miembros de las organizaciones sean conscientes de que sus acciones tienen consecuencias, no sólo para ellos sino también para la sociedad y el medio ambiente. Dentro de lo reconocido como el espíritu de la sociedad moderna globalizada, se hallan asuntos organizacionales que reclaman la atención y superación del reto ético. Esta sociedad se caracteriza por la deslocalización del trabajo, la apertura de mercados a escala global, la libre circulación de transacciones económicas y la desaparición de fronteras culturales, a raíz del aumento de flujos migratorios. Estos rasgos de la globalización demandan un abordaje ético práctico y universal en doble sentido: la definición de mínimos y máximos morales. Los mínimos tratan de normas morales, independientes de la cultura y establecidas por acuerdos intersubjetivos; los máximos, del reconocimiento, por parte de los miembros de una comunidad, de la necesidad de apropiar el derecho a la diferencia y de buscar el ideal del buen vivir.

Para efectos de la contextualización, cabe reseñar los planteamientos que formularon los integrantes de la Escuela de Frankfurt sobre los retos que en su momento debía enfrentar la sociedad, y que persisten sin solución satisfactoria desde que fueron evidenciados por Platón (1978). Algunos retos relevantes son el carácter totalitario de la sociedad, la instrumentalización del ser humano, la asunción del mundo material sobre el espiritual, que conduce al alejamiento de las personas de su género humano (Carmona, 2016) y la ruptura de las relaciones sociales por la notable ausencia de reconocimiento (Adorno, 2001). Esta denuncia de la sociedad moderna sentó las bases para que la lógica económica y social fuera puesta bajo observación crítica, con miras al desarrollo de un proyecto de vida humano más satisfactorio, donde la instrumentalización de la razón cediera su lugar a la razón como autonomía (Horkheimer y Adorno, 1969).

Una propuesta, de poca fidelidad con la Escuela de Fankfurt, es la ética discursiva de Habermas (1994), el reto ético tendría que ser asumido por una ética mínima, que pone de manifiesto la fundamentación de la validez de los enunciados y juicios morales a través de los presupuestos del discurso. Otras propuestas no frankfurtianas son las de Ernst Tugendhat, Judith Butler y Salvador Giner, quienes han dedicado sus estudios al develamiento del origen de la moral a partir de un estudio de sociogénesis que corrobore los límites interpretativos del análisis propuesto por el determinismo biológico en torno a la moral. En el marco social advertido, se inserta la ética organizacional como una manera de aplicar la ética en la dirección y operación de aquellos constructos humanos que permiten satisfacer necesidades, es decir, las organizaciones. Desde finales del siglo XX se ha hecho más imprescindible su aplicación, cuando se hicieron públicos fraudes financieros de grandes organizaciones, debido a actuaciones de directivos y empleados, en las que intereses individuales se pusieron por encima de intereses grupales, sociales y medio ambientales (Soltani, 2014). Fortalecer la ética en las organizaciones se ha hecho urgente, no sólo para responder a las reglamentaciones impuestas por parte de los estados, como la ley Sarbanes Oxley en Estados Unidos (Treviño et al., 2004), sino también por la presión de una sociedad que exige prácticas éticas (Padilla et al., 2017).

En respuesta a lo anterior, algunas organizaciones han implementado programas de ética, entendidos como un conjunto de políticas y actividades, cuyo objetivo principal es fomentar comportamientos éticos en una organización. Empero, aunque estos programas son una iniciativa prometedora, no es suficiente con su adopción. MacLean et al. (2015) plantean que los programas simbólicos o superficiales pueden hacer más daño que bien, por ello se necesita idear métodos que permitan mejorar tanto su eficiencia como su eficacia (Weber y Wasieleski, 2013). Es decir, estos programas deben cumplir los objetivos para los que fueron creados, para lo cual se requiere identificar lo que se está haciendo bien y lo que se puede mejorar. Sin embargo, aunque se ha reconocido su importancia, existen pocos estudios sobre la evaluación de programas de ética en las organizaciones (Kaptein, 2015). En el caso de las universidades, como organizaciones cuya naturaleza académica plantea continuas reflexiones sobre el comportamiento, se necesita un compromiso con la ética y la educación ética, aspectos que deben estar integrados dentro de su cultura (Weber, 2006; Toro y Rodríguez, 2017). Una forma de lograrlo, es a través de la implementación de programas de ética organizacional, ya que éstos tienen componentes que pueden contribuir tanto en la formación como en el desarrollo de culturas éticas.

Aunque algunos estudiosos han tratado de investigar sobre la eficacia de las iniciativas de ética en las instituciones de educación superior, se debe señalar la delgadez de ese cuerpo de literatura. De hecho, las búsquedas bibliográficas realizadas en las herramientas Scopus y Web of Science, entre los años 2015 y 2017, han permitido constatar que existe poca información sobre evaluación de programas de ética en universidades. Esto muestra que hay un vacío en el conocimiento donde se puede aportar con investigaciones, lo cual es la primera razón de ser de este artículo. La segunda razón es que se desea 
aprovechar el antecedente empírico de la Universidad Nacional de Colombia (UN), institución que tuvo, entre los años 2008 y 2012, un programa de ética denominado Sistema de Gestión del Entorno Ético (SEG), con su respectiva evaluación llamada Observatorio del Entorno Ético (OEE).

El presente artículo propone un modelo para la evaluación de programas de ética organizacional en universidades, considerando los avances teóricos y los antecedentes empíricos de la UN. Para ello, se parte de una discusión teórica sobre los programas de ética y su evaluación. A continuación, se describe la metodología para la elaboración del modelo. Luego se presentan y discuten los resultados de la investigación. Finalmente se exponen las principales conclusiones.

\section{PROGRAMAS DE ÉTICA ORGANIZACIONAL}

Los programas de ética organizacional pueden ser definidos como los sistemas de control ético formales o tangibles de la organización (Treviño et al., 2004; Kaptein, 2015). Se componen de código de ética, formación en ética, responsables de prácticas éticas y canales éticos. Pueden existir programas de ética con componentes adicionales, pero los anteriores son los que comúnmente se citan en la literatura (Treviño et al., 2004; Etebarian y Yazdi, 2016). Estos programas promueven el desarrollo de la sensibilidad ética, facilitan la promoción de valores éticos, promueven la toma de decisiones éticas y son de importancia estratégica, incluso si no está en juego la imagen de la organización. Su implementación proporciona guías para el comportamiento ético y ayuda a comunicarlas para que las personas sean conscientes de lo que se espera de ellas (Yeshu, 2016).

Las universidades deben implementar programas de ética, ya que no sólo necesitan generar conocimientos, hacer proyección social y formar profesionales íntegros, sino también luchar contra la corrupción (Sedigh, 2017). Estas instituciones requieren promover ambientes éticos para que sus miembros estén guiados por valores y puedan contribuir al logro de sus fines misionales (Osuna y Luna, 2011). Las universidades, como lo afirman Toro y Rodríguez (2017), "tienen la responsabilidad de dar a conocer a sus estudiantes los problemas éticos con que se enfrentarán en el mundo laboral y deben asegurarse que la enseñanza de la ética organizacional sea una parte integral del currículo de cualquier profesión" (p.168).

Sin embargo, cuando los programas de ética se adoptan como una manera de esconder fallos, ganar buena imagen o protegerse frente a futuras investigaciones, pueden hacer más daño que bien. Esto se debe a que cuando los empleados notan un desacoplamiento entre el programa y su cumplimiento real, puede haber una tendencia al comportamiento poco ético. Para evitar la percepción de desajuste, es necesario el compromiso de la alta dirección para predicar con el ejemplo y crear políticas y prácticas que permitan alinear los comportamientos reales con los objetivos del programa de ética, a la vez que se tienen sistemas de monitoreo del desempeño que identifiquen las faltas a la ética.

\section{Evaluación de programas de ética organizacional}

La evaluación de un programa de ética consiste en un seguimiento continuo, que permita verificar si se están cumpliendo los objetivos propuestos, tanto en términos de eficiencia como de eficacia. Es decir, consiste en revisar que los recursos asignados se estén usando bien y que las actividades ejecutadas sean de calidad, a la vez que se monitorea su impacto en el comportamiento de los miembros de la organización (Andreisová, 2016; Ferrell et al., 2016; Gazley et al., 2016). Desafortunadamente, la mayor parte de las organizaciones que tienen programas de ética, sólo realizan evaluaciones de eficiencia, dejando de lado la preocupación por el efecto en el comportamiento. Al respecto, el Departamento de Comercio de los Estados Unidos (2007) sostiene que es irresponsable dedicar atención y recursos a un programa sin evaluar su eficacia, para determinar si los esfuerzos valen la pena.

Por tanto, la evaluación de un programa de ética debe basarse no sólo en evidencia que dé cuenta de su eficiencia, sino también en el diálogo con los grupos de interés. Siendo ellos los que están en contacto directo con las actividades que se realizan, al conocer sus percepciones es posible identificar el impacto de lo que se está haciendo. La legitimidad de la organización depende de su capacidad para cumplir con las expectativas de sus grupos de interés (Freeman, 1994). Además, al ser personas (naturales o jurídicas) que tienen un interés, un reclamo o una expectativa en las operaciones y decisiones de la organización, es un deber moral considerar la forma en que afectan o son afectadas por el desarrollo del objeto social de la institución (Vieira, 2013) y actuar conforme a esto.

A pesar de su importancia, en la revisión de la literatura sólo se encontraron dos estudios que tienen relación con la evaluación de programas de ética. El primero buscaba evaluar el programa de ética de General Dynamics (Barker, 1993), para lo cual hicieron excavación documental, entrevistas, encuestas, observación participante, observación de los medios de comunicación y corroboración con miembros de la organización. Los resultados revelaron que el programa fue exitoso en el logro de los objetivos específicos (comunicar 
reglas y normas de conducta, mejorar la imagen de la empresa y seguir ganando contratos del gobierno), pero no exitoso en el logro de objetivos más amplios (mejorar la calidad de vida laboral a través del ambiente de trabajo, donde se propiciara el respeto mutuo, además de promover un trato justo a los empleados por parte de los gerentes y supervisores). Los medios utilizados para recolectar la información permitieron una evaluación amplia del programa de ética, debido a su variedad y al contacto directo con los implicados, por lo que fueron tenidos en cuenta para el modelo que se propone en el presente artículo.

El segundo buscaba evaluar los programas de ética de seis grandes compañías de Estados Unidos (Treviño et al., 1999), para lo cual se hizo una encuesta a 10.000 de sus empleados. La evaluación se realizó con base en los siguientes criterios: 1) comportamientos no éticos observados en el último año, 2) sensibilidad demostrada por los empleados en asuntos éticos o legales en el trabajo, 3) búsqueda de consejos para un mejor cumplimiento ético, 4) facilidad para informar a la dirección sobre asuntos éticos, 5) informes de la organización sobre transgresiones a la ética, 6) percepción de mejora de la calidad ética en la toma de decisiones debido a los programas desarrollados y 7) sentido de compromiso del empleado con la organización. Esta evaluación permitió comparar los resultados con evaluaciones anteriores y evidenciar los cambios que se presentaron a partir de la implementación de los programas. Los criterios utilizados fueron tenidos en cuenta para el modelo propuesto en el presente artículo.

Existen otras iniciativas para evaluar programas de ética como la auditoría ética y la auditoría social (ver Tabla 1). La auditoría ética evalúa el monto del daño o beneficio resultante de las operaciones de la organización, teniendo en cuenta sus niveles: estratégico, táctico y operativo, con el fin de determinar si se está actuando de acuerdo con parámetros morales. Además de revelar las diferentes formas en que la organización puede hacer daño a sus grupos de interés, debe incluir la respuesta a ese daño y establecer medidas para evitar daños futuros (Ostapski y Pressley, 1992). La auditoría social es definida como la evaluación sistemática, objetiva y periódica de las actividades sociales de la organización (Ruiz y Retolaza, 2005). Además, se analiza la forma en que la organización responde a las exigencias de los grupos de interés, referentes a su responsabilidad social. La búsqueda de la mejora en sus actuaciones sociales y la transparencia constituyen los objetivos prioritarios perseguidos.

Tabla 1: Resumen auditoría ética y auditoría social

\begin{tabular}{|c|l|l|l|}
\hline Iniciativa & \multicolumn{1}{|c|}{ Objetivo } & \multicolumn{1}{c|}{ Aportes } & \multicolumn{1}{c|}{ Limitaciones } \\
\hline $\begin{array}{c}\text { Auditoría } \\
\text { ética }\end{array}$ & $\begin{array}{l}\text { Evaluar los efectos de las } \\
\text { actividades de la } \\
\text { organización en términos } \\
\text { morales. }\end{array}$ & $\begin{array}{l}\text { Permite hacer un diagnóstico del } \\
\text { impacto moral de las actividades } \\
\text { de la organización, así como la } \\
\text { respuesta a dicho impacto. }\end{array}$ & $\begin{array}{l}\text { Las personas la relacionan con control } \\
\text { y sanciones. }\end{array}$ \\
\hline $\begin{array}{c}\text { Auditoría } \\
\text { social }\end{array}$ & $\begin{array}{l}\text { Evaluar el impacto social de } \\
\text { las actividades de la la } \\
\text { organización. }\end{array}$ & $\begin{array}{l}\text { Permite analizar la respuesta de la } \\
\text { organización a temas relacionados } \\
\text { con la responsabilidad social. }\end{array}$ & $\begin{array}{l}\text { Se enfoca en la responsabilidad social } \\
\text { y no tien en cuentica otras dimensiones } \\
\text { de lacional. }\end{array}$ \\
\hline
\end{tabular}

A pesar de que la auditoría ética ha logrado avances, no ha funcionado en todo tipo de organizaciones, puesto que se relaciona con aspectos coercitivos y se genera la impresión de que se están buscando errores. En el caso de la auditoría social tampoco se han logrado avances, debido a las complejas metodologías diseñadas para medir el cumplimiento de la responsabilidad social. Por esta razón, dependiendo del tipo de organización, es necesario analizar la mejor forma de evaluar las iniciativas éticas. Tal es el caso de las universidades, donde son más pertinentes las evaluaciones con un carácter reflexivo y dialógico, que sean coherentes con la naturaleza y principios de este tipo de instituciones.

\section{METODOLOGÍA}

La investigación de la cual se desprende este artículo tuvo un enfoque cualitativo. Este es útil cuando es necesario apoyar los hallazgos teóricos con experiencias reales, porque logra un acercamiento a la realidad que se estudia a través de la interacción entre el investigador y los investigados (Sandoval, 1996). Por esta razón, se tuvo en cuenta la experiencia de la UN y se dialogó con las personas que hicieron parte del SEG y del OEE, ya que de esta manera se podía obtener información útil que aportara ideas para la construcción del modelo. El tener contacto con las personas implicadas en el proceso permitió obtener información adicional a la que se encontraba en registros o informes y conocer de primera mano sus percepciones sobre los aciertos y errores de las evaluaciones que se llevaron a cabo. Las etapas que se siguieron en la investigación se muestran en la Figura 1. 


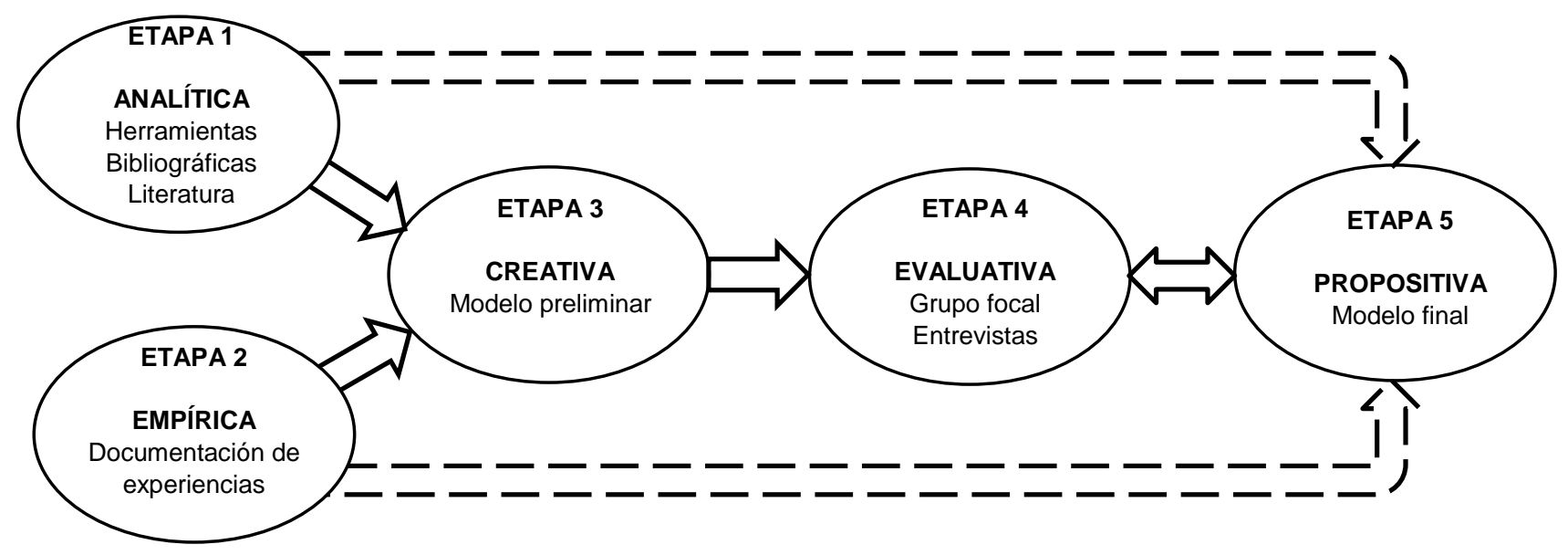

Fig. 1: Metodología para la construcción del modelo para la evaluación de los programas de ética en universidades

La etapa analítica empezó con la revisión de la literatura a través de las herramientas bibliográficas Scopus y Web of Science, la cual se resume en la Tabla 2.

Tabla 2: Resultados de la revisión de la literatura

\begin{tabular}{|c|c|c|c|}
\hline Herramienta bibliográfica & Ecuaciones de búsqueda & $\begin{array}{c}\text { Cantidad de documentos } \\
\text { encontrados }\end{array}$ & $\begin{array}{c}\text { Documentos } \\
\text { pertinentes }\end{array}$ \\
\hline Scopus & $\begin{array}{c}\text { ("Ethic* program*) AND } \\
\text { (university or college or academi* or } \\
\text { institute) }\end{array}$ & 12 & 1 \\
\hline Web of Science & $\begin{array}{c}\text { ("Ethic* program*) AND } \\
\text { (evaluation) }\end{array}$ & 31 & 1 \\
\hline Scopus & & 7 & 0 \\
\hline Web of Science & & 21 & 1 \\
\hline
\end{tabular}

Para la clasificación de los documentos como pertinentes o no, se utilizó el criterio de su relación directa con los temas que se abordaban en la investigación. De los documentos pertinentes que aparecen en la Tabla 2 , la primera ecuación arrojó el mismo resultado en las dos herramientas, el artículo de Weber (2006). En éste se abordan aspectos relacionados con la implementación de un programa de ética en la Universidad de Duquesne, incluyendo: declaración de la misión, compromiso del liderazgo con la ética, cursos de ética, código de ética, oficina de ética y evaluación de la iniciativa ética. El autor plantea la importancia que representan las lecciones aprendidas para otras universidades que deseen implementar un programa de ética. Sin embargo, este artículo no profundiza en su evaluación. La segunda ecuación arrojó como resultado el artículo de Barker (1993), reseñado en el apartado anterior.

La búsqueda sistemática se complementó con búsquedas narrativas en las principales revistas sobre ética organizacional, como Journal of Business Ethics y Business Ethics Quarterly, indagando por: programas de ética, evaluación de programas de ética, programas de ética en universidades y evaluación de programas de ética en universidades. Adicionalmente se consultaron otro tipo de textos sobre los temas mencionados. Estas revisiones permitieron verificar que existe un vacío en el conocimiento sobre la evaluación de programas de ética en universidades (Weber, 2006; Kaptein, 2015), ya que durante el período de consultas (febrero de 2015 a abril de 2017) no se encontraron investigaciones que abordaran directamente el tema.

Posteriormente, en la etapa empírica se recurrió al antecedente de la UN. Esto incluyó la revisión de documentos y la realización de entrevistas semiestructuradas a tres de los encargados de diseñar e implementar el OEE, a quienes se les indagó por: cómo surgió, en qué fuentes se basaron para diseñarlo, las actividades que se realizaron, lo que funcionó y no funcionó, las reacciones de los miembros de la Universidad frente a los informes generados por el OEE y sus percepciones sobre aspectos adicionales. La información recolectada permitió documentar el caso y de esta manera describir y comprender mejor la experiencia de los encargados del OEE. El desarrollo de esta etapa también permitió reconocer los medios utilizados para recolectar la información (excavación documental, entrevistas, encuestas y monitoreo de medios), los cuales se tuvieron en cuenta en el modelo propuesto. 
En la etapa creativa se extrajeron elementos de la etapa uno, específicamente se tomaron de la investigación de Barker (1993) los instrumentos de recolección de información. Del estudio de Treviño et al. (1999) se tuvieron en cuenta los criterios utilizados para la evaluación y se integraron en el modelo de entrevista sugerido (el cual no se presenta en este artículo por cuestiones de espacio). Estos insumos se complementaron con la documentación y análisis del caso de la UN, lo que permitió articular la información teórica con la información empírica para aplicarla al contexto de las universidades y proponer la primera versión del modelo.

En la etapa evaluativa se validó la versión inicial del modelo en dos momentos. Primero, se realizó un grupo focal con cuatro de los encargados del OEE, a quienes se les presentó el modelo y se les pidió opinar sobre cada uno de sus componentes, la factibilidad de aplicarlo, las fortalezas, las debilidades y, en general, recomendaciones para su mejoramiento. Segundo, se realizaron entrevistas a tres miembros del OEE, a quienes se les expuso el modelo corregido de acuerdo a las sugerencias del grupo focal.

En la etapa propositiva se efectuó un proceso analítico-interpretativo, que se apoyó en las etapas anteriores, para identificar otras oportunidades de mejoramiento. Se hicieron los cambios necesarios y se propuso la versión final del modelo, el cual fue validado por cuatro expertos en un último grupo focal. "El juicio de expertos se define como una opinión informada de personas con trayectoria en el tema, que son reconocidas por otros como expertos cualificados en éste y que pueden dar información, evidencia, juicios y valoraciones" (Escobar y Cuervo, 2008, p.29). Los cuatro participantes en el último grupo focal pertenecen al grupo de investigación Ética Empresarial y Empresariado Social - Ethos, tienen estudios de maestría y doctorado, son investigadores activos en ética organizacional y tienen experiencia en programas de ética y su evaluación. A partir de las recomendaciones emanadas del último grupo focal, se consolidó la versión final de la propuesta que se expone en el siguiente apartado.

\section{RESULTADOS}

Como resultado de las etapas 1 y 2, se documentó y analizó la experiencia del OEE en la UN. Éste fue planteado en el 2008 para monitorear la implementación del SEG. Cada año se recolectó y analizó información que permitía evaluar la eficiencia y eficacia del SEG. La evaluación se hacía en tres frentes: primero, la forma en que se estaban aplicando los recursos asignados al SEG; segundo, la calidad de las actividades ejecutadas y; tercero, la percepción de profesores, estudiantes y administrativos sobre la aplicación de los valores declarados en el Compromiso Ético de la Institución. Para ello se hizo excavación documental, entrevistas, encuestas y monitoreo de medios de comunicación (Rodríguez et al., 2012). Los informes presentados por el OEE tuvieron una amplia difusión y acogida por los miembros de la UN, quienes expresaron su acuerdo con lo planteado en él. Esto sirvió, no sólo para evaluar la eficiencia y eficacia del SEG, sino también para tomar medidas que permitieran corregir algunos de los fallos éticos detectados. Sin embargo, el OEE tuvo debilidades, algunas de las cuales se relacionan en la Tabla 3 , con la respectiva solución en el modelo que se propone.

Tabla 3: Resultado análisis hermenéutico

\begin{tabular}{|l|l|}
\hline \multicolumn{1}{|c|}{ Debilidades encontradas en el OEE } & \multicolumn{1}{|c|}{ Cómo se solucionan en el modelo propuesto } \\
\hline $\begin{array}{l}\text { Se hacía énfasis principalmente en los } \\
\text { valores del Compromiso Ético. }\end{array}$ & $\begin{array}{l}\text { Los valores del Compromiso Ético siguen siendo importantes, pero } \\
\text { también se tienen en cuenta los demás componentes del programa de } \\
\text { ética. }\end{array}$ \\
\hline $\begin{array}{l}\text { Volumen de información muy alto. } \\
\text { La información obtenida se puede analizar de manera segmentada por } \\
\text { cada componente del programa de ética, para que el proceso sea más } \\
\text { estructurado y el volumen de información más manejable. }\end{array}$ \\
\hline $\begin{array}{l}\text { Los informes eran difíciles de elaborar por } \\
\text { la cantidad de información recolectada, } \\
\text { principalmente la proveniente de } \\
\text { entrevistas. }\end{array}$ & $\begin{array}{l}\text { Las entrevistas y demás instrumentos están segmentados en los } \\
\text { componentes del programa de ética para facilitar su análisis. } \\
\text { Adicionalmente, se propone el uso de herramientas de análisis cualitativo } \\
\text { (como el Atlas.ti) para hacer que este proceso sea más eficiente. }\end{array}$ \\
\hline $\begin{array}{l}\text { Se hacía un año sólo los análisis de } \\
\text { eficiencia y otro año sólo los de eficacia. }\end{array}$ & $\begin{array}{l}\text { En el modelo propuesto se tienen en cuenta los dos componentes } \\
\text { (eficiencia y eficacia) simultáneamente. }\end{array}$ \\
\hline $\begin{array}{l}\text { Los nombres de los elementos del } \\
\text { programa de ética no tenían una afiliación } \\
\text { a nivel teórico. }\end{array}$ & $\begin{array}{l}\text { Se utiliza un lenguaje común en la literatura académica sobre programas } \\
\text { de ética y su evaluación. }\end{array}$ \\
\hline $\begin{array}{l}\text { El OEE no estaba configurado como un } \\
\text { modelo. }\end{array}$ & $\begin{array}{l}\text { Se documentó la experiencia y con aportes adicionales se propuso el } \\
\text { modelo que se explica más adelante. }\end{array}$ \\
\hline
\end{tabular}


El modelo propuesto para la evaluación de programas de ética organizacional en universidades se muestra en la Figura 2. Éste se compone de dos partes, la primera (eficiencia) da cuenta de la forma en que se han aplicado los recursos asignados al programa de ética, la calidad de las actividades realizadas y la participación de los principales grupos de interés: responsables de la gestión de la investigación, académicos, grupos de investigación, empresarios, estudiantes (Vieira, 2013), egresados y administrativos. La segunda (eficacia) permite determinar el impacto del programa de ética, evaluando si se lograron los objetivos propuestos y los efectos que se han tenido después de su implementación. En este sentido, se indaga por aspectos como conocimiento del código de ética y su aplicación en las labores diarias, conocimiento de los valores institucionales y cómo se viven en la realidad, opiniones acerca del programa de ética y los cambios que se perciben en el comportamiento, importancia que le da la universidad a la formación en ética y satisfacción con la gestión ética de la universidad. Para obtener la información se utilizan instrumentos de corte cualitativo y cuantitativo.

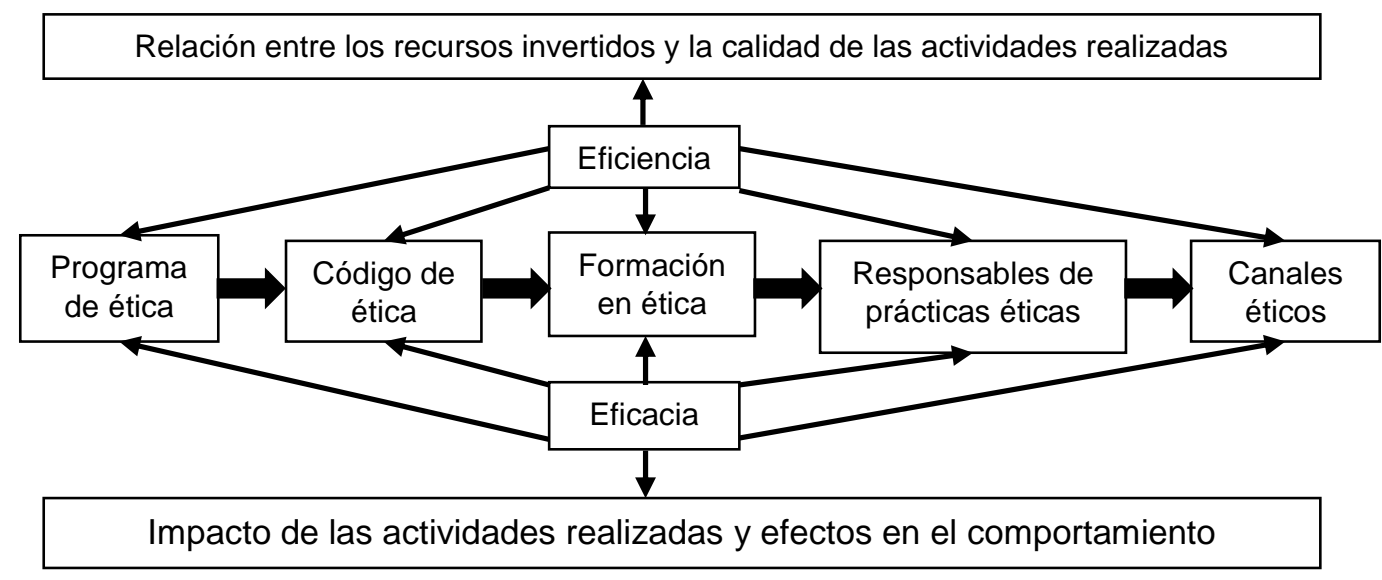

Fig. 2: Modelo propuesto para la evaluación de programas de ética organizacional en universidades

La forma de aplicación del modelo se muestra en la Figura 3 y sus fases se explican a continuación.

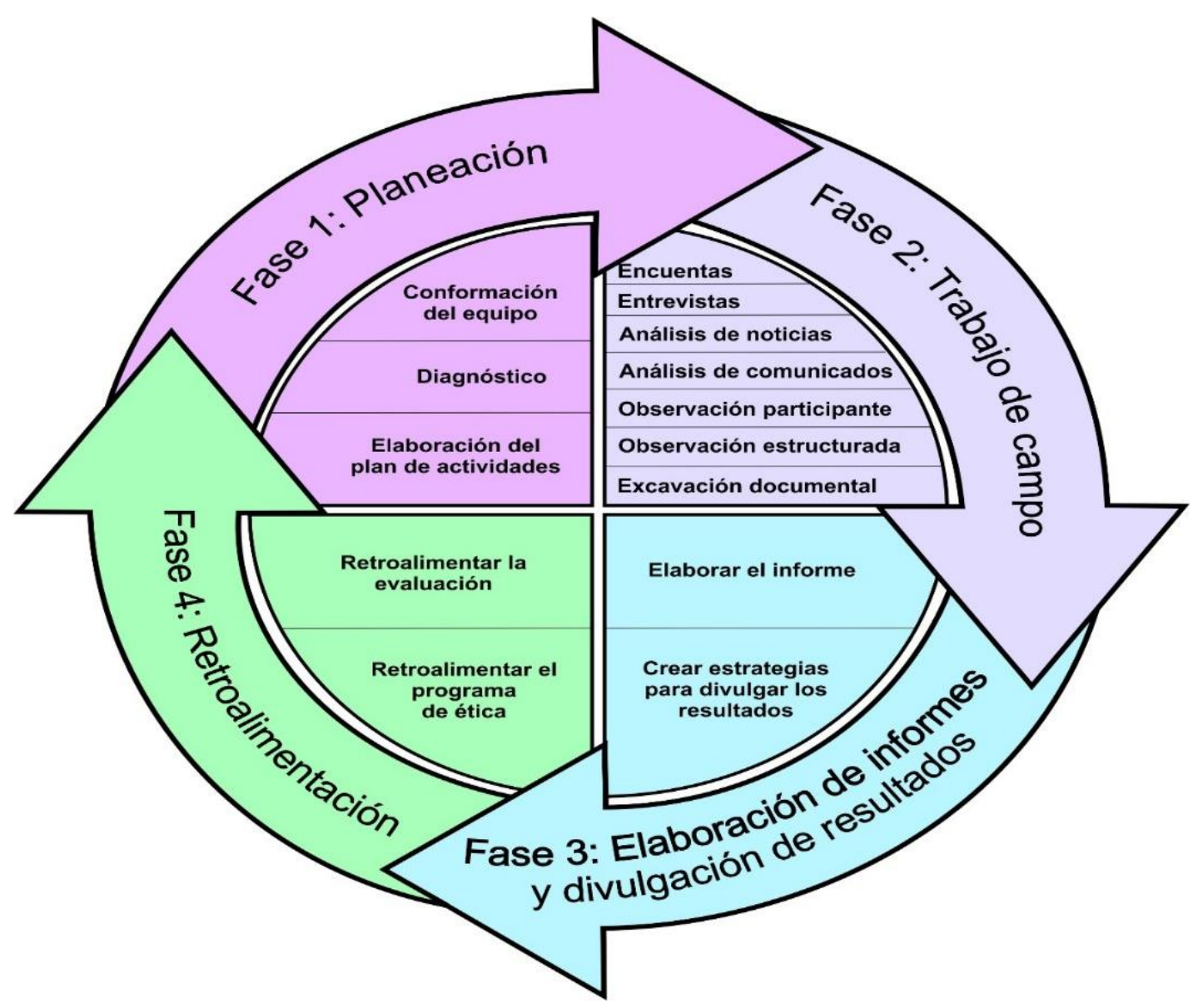

Fig. 3: Metodología para la implementación del modelo propuesto 


\section{Fase 1. Planeación}

La primera actividad de esta fase es la conformación del equipo, que consiste en elegir a las personas que se encargarán de llevar a cabo la evaluación, de acuerdo con las cualidades y habilidades que se requieren en el proceso. La cantidad de integrantes dependerá de factores como el tamaño y configuración de la universidad. Se recomienda tener en cuenta las siguientes características: 1) Que al menos uno haya estado presente en la implementación del programa de ética. 2) Que provengan de diferentes estamentos y niveles de la universidad. 3) Que al menos dos pertenezcan a grupos de interés externos. Un equipo así conformado puede aportar diversidad en las visiones de los fenómenos estudiados, tanto desde el conocimiento de la universidad y su cultura, como desde la imparcialidad, objetividad y experiencia provenientes de otros contextos.

La segunda actividad es el diagnóstico, con el que se busca establecer una línea de base, indagando por las actividades que se han realizado para ajustar la evaluación a las características de la universidad. Aquí se pueden utilizar diferentes instrumentos de recolección de información, como excavación documental, encuestas, entrevistas, grupos focales y otros que se consideren convenientes; involucrando a los encargados de implementar el programa de ética y a personas que tengan información acerca de las actividades que se han desarrollado como parte de éste.

La tercera parte es la elaboración del plan de actividades, la cual consiste en definir las acciones que se van a llevar a cabo para evaluar el impacto del programa de ética, teniendo en cuenta el diagnóstico. También se asignan funciones de acuerdo a las habilidades de cada integrante, se apropian los recursos y se define el alcance de la evaluación, especialmente en cuanto a los grupos de interés que son prioritarios para la universidad. Es necesario seleccionar las fuentes de información tanto primarias como secundarias y socializar con los grupos de interés elegidos lo que se va a hacer, por qué y para qué.

\section{Fase 2. Trabajo de campo}

Entrevistas: permiten conocer la percepción que tienen los miembros de la universidad acerca del programa de ética y de cada uno de sus componentes. Esto se logra mediante el diálogo con miembros de la universidad como: representantes (profesorales y estudiantiles), rector, directores de área, decanos, secretarios de facultad, jefes de departamento y miembros de sindicatos. Para facilitar el análisis, se recomienda que las preguntas estén segmentadas por cada componente del programa de ética, indagando por las percepciones que tienen acerca de las actividades desarrolladas, si se sienten identificados con la forma en que se aborda, si creen que es necesario realizar ajustes y otras preguntas que faciliten el entendimiento del impacto que se ha tenido con el desarrollo del programa de ética.

Encuestas: se usan para complementar la información recolectada por medio de entrevistas. Se propone que sean enviadas vía web a estudiantes, profesores y administrativos. Se pregunta en ellas por el conocimiento de los componentes del programa, la asistencia a las actividades, si han percibido cambios positivos en los comportamientos después de implementar el programa. Se recomienda que las preguntas puedan responderse en escalas tipo Likert y que se incluyan dos preguntas abiertas, una que permita al encuestado dar su opinión sobre el programa y otra que recoja sus sugerencias de mejora.

Análisis de noticias: permite conocer los aspectos que se destacan por parte de medios masivos de comunicación. Se identifican los medios de comunicación oficiales de la universidad y los principales medios nacionales e internacionales y se realiza un análisis a las noticias que tienen que ver con el programa de ética y otras iniciativas para fomentar la ética. Se recomienda que el monitoreo sea constante. En las noticias seleccionadas se debe analizar el contenido, analizando aspectos positivos o negativos e identificando argumentos.

Análisis de comunicados oficiales y no oficiales emitidos por los grupos de interés: a través de estos se puede conocer en qué situaciones las personas utilizan el discurso ético y los aspectos que allí se resaltan, así como la apropiación de los valores institucionales en los discursos que emiten. También se debe prestar atención a las discusiones que surjan en torno a la ética y las alertas ante faltas éticas. Para el análisis se seleccionan comunicados donde se aborden problemáticas de la vida universitaria como actas, cartas y correos electrónicos que sean relevantes en el período que se realiza la evaluación.

Observación participante: posibilita que se analicen ciertos aspectos en contextos reales. Aquí se propone la realización de una actividad semestral por parte del grupo evaluador. Por ejemplo, una jornada de trueque, una iniciativa de voluntariado, una brigada de prevención de problemas comunes y, en general, aquello que permita poner en práctica valores morales. El equipo evaluador analizará el comportamiento de los miembros de los grupos de interés en función de la práctica de los valores institucionales. 
Observación estructurada: permite analizar el comportamiento de los miembros de los grupos de interés en el desarrollo del programa de ética. De acuerdo a esto, se eligen algunas actividades y se definen los parámetros que guiarán la observación y permitirán evaluar su impacto. Por ejemplo, el nivel de asistencia, la receptividad, la interacción y la respuesta en los momentos que se requiere intervención. Aquí es importante dialogar con algunos participantes para saber las opiniones que tienen acerca de la actividad, lo que piensan acerca de su utilidad o las sugerencias que tienen.

Excavación documental: Por una parte, se revisan los registros de los componentes del programa. Por ejemplo, denuncias realizadas a través del canal ético, analizando qué tipo de denuncias hacen, cómo se han resuelto y qué seguimiento se les ha dado. Se revisan los documentos financieros del programa, para dar cuenta de la ejecución presupuestal. También se recurre a las evaluaciones de cada una de las actividades del programa, lo que permite dar un concepto sobre la calidad de las mismas. Por otra parte, se realiza una búsqueda sobre investigaciones, trabajos de grado y tesis en torno a la ética. Esto indicará qué tan dispuestos están los estudiantes y profesores a profundizar en ella. Adicionalmente, esto dará a conocer los aportes que se están haciendo en el área. Asimismo, se puede revisar la oferta de asignaturas relacionadas con la ética, lo que proporcionará una visión de la formación en ética de los estudiantes.

\section{Fase 3. Elaboración del informe y divulgación de resultados}

En esta fase se elabora un documento que exponga la información de manera clara y coherente. Una primera parte dará cuenta de la evaluación de eficiencia, mencionando, para cada elemento y para el programa de ética: la relación entre los recursos invertidos y la calidad de las actividades desarrolladas y la respuesta de los grupos de interés a dichas actividades. Se recomienda que el análisis de esta parte del informe sea de tipo cuantitativo. La segunda parte tendrá una estructura basada en cada componente del programa, es decir: código de ética, formación en ética, responsables de prácticas éticas, canal ético y un apartado final con las percepciones acerca del programa. Se recomienda el uso de software para el análisis cualitativo ya que, según la experiencia del OEE, esta es la fase más dispendiosa del proceso, por lo que es útil disponer de herramientas que ayuden a una mejor interpretación de la información recolectada.

De cada componente se puede exponer: los resultados de la evaluación de eficiencia, percepciones de los grupos de interés, cambios que se han suscitado en el comportamiento de las personas, aspectos positivos y aspectos que se deben mejorar. Cuando se hayan realizado varias evaluaciones, es necesario incluir un análisis de la evolución del componente. Se recomienda apoyar los resultados con material multimedia como videos, presentaciones y fotos. También se deben crear estrategias que permitan la presentación de los resultados de una forma atractiva ante los grupos de interés. Se requiere una amplia divulgación para que los hallazgos sean conocidos por todos los involucrados en el proceso de evaluación, siendo indispensable realizar jornadas de socialización que lleven al diálogo, la reflexión y el debate.

\section{Fase 4. Retroalimentación}

Esta fase cierra el ciclo del modelo propuesto, dando inicio a una nueva evaluación. Sus principales insumos son los informes de evaluación y las conclusiones de las discusiones llevadas a cabo en las jornadas de difusión. A partir de allí, se discuten los hallazgos con los encargados del programa de ética para que ellos: a) realicen ajustes al programa y a cada uno de sus componentes, de tal manera que se logre un mejoramiento continuo en términos de eficiencia y eficacia; b) informen a las instancias respectivas sobre las debilidades éticas detectadas y promuevan la implementación de soluciones y; c) informen a las instancias respectivas sobre las fortalezas éticas detectadas para que se analice qué es lo que está funcionando bien para continuar haciéndolo y, a la vez, se hagan reconocimientos públicos que se constituyan en un premio para los protagonistas y un ejemplo para los grupos de interés.

Además, a lo largo de todo el proceso de evaluación es posible identificar lo que está funcionando correctamente y lo que es necesario corregir, respecto a la propia evaluación. Se recomienda, por tanto, que se lleve un diario de campo que dé cuenta de los aciertos y desaciertos de dicho proceso. La información allí escrita permitirá tomar las acciones necesarias para que las evaluaciones funcionen cada vez mejor. La retroalimentación es útil en todas las etapas del modelo. Por ejemplo, en el trabajo de campo, se deben refinar los instrumentos de recolección de información para garantizar la participación de los grupos de interés seleccionados. Asimismo, en el análisis de la información, dado el alto volumen que se recolecta, es menester continuar actualizando los mecanismos para su tratamiento y disponer de herramientas tecnológicas de vanguardia. 


\section{DISCUSIÓN}

Como se ha mencionado a lo largo del documento, diversos autores han identificado la necesidad de evaluar los programas de ética organizacional (Andreisová, 2016; Ferrell et al., 2016; Gazley et al., 2016). Existen formas variadas de llevar a cabo dicha evaluación, unas se centran en la eficiencia, otras en la eficacia y otras en la combinación de ambas. Las primeras buscan poner a prueba la forma en que los recursos asignados son ejecutados y la calidad de las actividades realizadas; las segundas dan cuenta del impacto que dichas actividades tienen en el comportamiento de los miembros de la organización. Mientras que los estudios de Barker (1993) y Treviño et al. (1999) se centraron en la eficacia, en el presente artículo se propone un modelo mixto que evalúa la eficiencia y la eficacia. Esto dado que es necesario hacer un control de la ejecución presupuestal, que permita informar a la alta dirección sobre la forma en que se están usando los recursos asignados y, a su vez, dar información sobre el impacto del programa de ética en cuanto a los cambios positivos en el comportamiento ético y el fortalecimiento de la cultura organizacional ética.

Sin embargo, para la propuesta del modelo se tuvieron en cuenta los retos éticos planteados por la escuela de Frankfurt y, en especial, el concerniente a la necesidad de un proyecto de vida más humano, en el que la instrumentalización de la razón cediera su lugar a la razón como autonomía (Horkheimer y Adorno, 1969). Por ello, el enfoque cualitativo es el que mayor peso tiene en el modelo, ya que él permite conocer las percepciones de los miembros de los grupos de interés y, mediante un proceso interpretativo, tener una idea sobre los efectos que tienen las actividades de cada componente del programa en los comportamientos de los miembros de la organización. Además, la variedad de estos instrumentos permite cruzar la información para lograr una mejor comprensión del impacto que ha tenido el programa. Esto se corresponde con lo afirmado por el Departamento de Comercio de los Estados Unidos (2007), en cuanto a la necesidad de obtener información adecuada y conocimiento esencial, a través de mecanismos de recepción de comentarios y reacciones, para hacer evaluaciones de eficacia de los programas de ética. Muchos de estos instrumentos también fueron utilizados por Barker (1993) y por los encargados del OEE.

En este sentido y de acuerdo al artículo de Weber (2006), en el que describe la experiencia de la implementación del programa de ética en la Universidad Duquesne, la retroalimentación de los miembros de los grupos de interés es un elemento importante para mejorar continuamente el programa. Esto es confirmado por las personas que se encargaron de implementar y evaluar el programa de ética en la UN, quienes manifestaron que la información suministrada por académicos, estudiantes y administrativos, además de quienes ejecutan el programa, es fundamental tanto para encontrar fortalezas a aprovechar y debilidades a cambiar, como para comprender los efectos que el programa está teniendo en los grupos de interés y en la cultura organizacional.

\section{CONCLUSIONES}

Las organizaciones y entre ellas las universidades, deben responder a las expectativas de sus grupos de interés, quienes esperan que en el desarrollo de su objeto social consideren aspectos éticos que les permitan cumplir sus obligaciones, sin poner en riesgo los intereses de los públicos que afectan o son afectados por ellas. Consecuente con esto, es necesario que se comprometan con iniciativas éticas, pero no de manera superficial, sino siendo conscientes de los beneficios que representa incentivar reflexiones y comportamientos éticos en los miembros de la comunidad universitaria. De estas iniciativas se destacan los programas de ética organizacional, los cuales son implementados cada vez con más frecuencia para promover comportamientos adecuados.

Los programas de ética implementados en cualquier tipo de organización deben ser evaluados, teniendo en cuenta aspectos relacionados con la eficiencia y la eficacia. Estas evaluaciones permitirán conocer si se están satisfaciendo las expectativas éticas y sociales de los grupos de interés, o si es necesario realizar cambios que permitan mejorar el impacto de lo que se está haciendo.

El modelo propuesto da respuesta a la necesidad de evaluar los programas de ética organizacional en universidades. Dando cuenta de la eficiencia, basada en la ejecución presupuestal y la calidad de las actividades realizadas. También evaluando la eficacia, basada en el impacto, considerando las percepciones de los grupos de interés en cuanto a la apropiación del código ético y los valores morales, los cambios en las actitudes y comportamientos éticos y el fortalecimiento de la cultura organizacional ética.

\section{REFERENCIAS}

Adorno, T., Minima Moralia, 3ª edición, 1-255, Taurus, Madrid, España (2001)

Andreisová, L., Building and Maintaining an Effective Compliance Program, https://goo.gl/e9GZem, International Journal of Organizational Leadership (en línea), 5(1), 24-39 (2016) 
Barker, R.A., An evaluation of the ethics program at General Dynamics, doi: 10.1007/BF01686444, Journal of Business Ethics (en línea), 12, 165-177 (1993)

Carmona, L.A., Ética, cultura humanista y organizaciones, https://goo.gl/qgVRC7, ISSN: 2357-4933, NOVUM (en línea), 6, 73-82 (2016)

Departamento de Comercio de los Estados Unidos, Ética Comercial, 1-343, International Trade Administration, Washington, Estados Unidos (2007)

Escobar, J. y Cuervo, A., Validez de contenido y juicio de expertos: una aproximación a su utilización, https://goo.gl/jRDRwh, Avances en Medición (en línea), 6, 27-36 (2008)

Etebarian, A. y Yazdi, N.M., The relationship between institutionalization of ethics and quality of work life with managers' job satisfaction, doi: 10.3923/ibm.2016.1177.1182, International Business Management (en línea), $10,1177-1182(2016)$

Ferrell, O.C.; Fraedrich, J. y Ferrell, L., Business ethics: ethical decision making and cases, 11a edición, 1615, Cengage learning, Boston, Estados Unidos (2016)

Freeman, R.E., A stakeholder theory of the modern corporation, https://goo.gl/Vs3Edj, Ethical theory and business (en línea), 50, 38-48 (1994)

Gazley, A.; Sinha, A. y Rod, M., Toward a theory of marketing law transgressions, doi: 10.1016/j.jbusres.2015.05.004, Journal of Business Research (en línea), 69(2), 476-483 (2016)

Habermas, J., Conciencia moral y acción comunicativa, 1-219, Edición 62, Barcelona, España (1994)

Horkheimer, M. y Adorno, T., Dialéctica del lluminismo, 1-303, Editorial Sur, Buenos Aires, Argentina (1969)

Kaptein, M., The Effectiveness of Ethics Programs: The Role of Scope, Composition, and Sequence, doi: 10.1007/s10551-014-2296-3, Journal of Business Ethics (en línea), 132, 415-431 (2015)

MacLean, T.; Litzky, B. y Holderness, D., When Organizations Don't Walk Their Talk: A Cross-Level Examination of How Decoupling Formal Ethics Programs Affects Organizational Members, doi: 10.1007/s10551-014-2103-1, Journal of Business Ethics (en línea), 128(2), 351-368 (2015)

Ostapski, S.A. y Pressley, D.G., Moral Audit for Diabco Corporation, doi: 10.1007/BF00871993, Journal of Business Ethics (en línea), 11(1), 71-80 (1992)

Osuna, C. y Luna, E., Valores Éticos en la Formación Universitaria de las Áreas de Ciencias Naturales e Ingeniería y Tecnología, en el Contexto de la Sociedad del Conocimiento, doi: 10.4067/S071850062011000500005, Formación Universitaria (en línea), 4(5), 29-36 (2011)

Padilla, C.P.; Arévalo, D.X.; Bustamante, M.A. y Vidal, C.L., Responsabilidad Social Empresarial y Desempeño Financiero en la Industria del Plástico en Ecuador, doi: 10.4067/S0718-07642017000400012, Información Tecnológica (en línea), 28(4), 93-102 (2017)

Platón, Diálogos, 1-458, Porrúa, Ciudad de México, México (1978)

Rodríguez, M.P.; Salazar, V. y Correa, J.S., Sistema de Gestión del Entorno Ético (SEG). Componente: Observatorio del Entorno Ético, Documento del Grupo Ética Empresarial y Empresariado Social ETHOS (https://goo.gl/dKNpdq, acceso 14 de abril de 2017), No publicado (2012)

Ruiz, M. y Retolaza, J.L., La auditoría social como instrumento de posicionamiento de la economía solidaria, https://goo.gl/XixdYR, CIRIEC-España, Revista de Economía Pública, Social y Cooperativa (en línea), 53, 285-304 (2005)

Sandoval, C.A., Investigación cualitativa, 1-313, ARFO Editores, Bogotá, Colombia (1996)

Sedigh, A.K., Ethics: An Indispensable Dimension in the University Rankings, doi: 10.1007/s11948-016-97581, Science and Engineering Ethics (en línea), 23, 65-80 (2017) 
Soltani, B., The Anatomy of Corporate Fraud: A Comparative Analysis of High Profile American and European Corporate Scandals, doi: 10.1007/s10551-013-1660-z, Journal of Business Ethics (en línea), 120(2), 251-274 (2014)

Toro, J. y Rodríguez, M.P., Formación en Ética en las Organizaciones: Revisión de la Literatura, doi: 10.4067/S0718-07642017000200018, Información Tecnológica (en línea), 28(2), 167-180 (2017)

Treviño, L.K.; Brown, M.E. y Wall, S.J., Managing to Be Ethical: Debunking Five Business Ethics Myths, doi: 10.5465/AME.2004.13837400, Academy of Management (en línea), 18(2), 69-83 (2004)

Treviño, L.K.; Weaver, G.R.; Gibson, D.G. y Toffler, B.L., Managing ethics and legal compliance: What works and what hurts, doi: 10.2307/41165990, California Management Review (en línea), 41(2), 131-151 (1999)

Vieira, J.A., The socially responsible management in Colombian public universities. Case study: the research function in public universities at Manizales (Colombia), Tesis Doctoral, Université de Rouen France, Facultad de Ciencias de la Gestión (2013)

Weber, J., Implementing an organizational ethics program in an academic environment: the challenges and opportunities for the Duquesne University Schools of Business, doi: 10.1007/s10551-005-3970-2, Journal of Business Ethics (en línea), 65, 23-42 (2006)

Weber, J. y Wasieleski, D.M., Corporate Ethics and Compliance Programs: A Report, Analysis and Critique, doi: 10.1007/s10551-012-1561-6, Journal of Business Ethics (en línea), 112(4), 609-626 (2013)

Yeshu, M.S., Role of ethics in business, https://goo.gl/4U19xk, ISSN: 2349-2589, KAAV International Journal of Economics, Commerce \& Business Management (en línea), 3, 48-57 (2016) 